\title{
Frequent genetic aberrations in the cell cycle related genes in mucosal melanoma indicate the potential for targeted therapy
}

Longwen Xu, Zhiyuan Cheng, Chuanliang Cui, Xiaowen Wu, Huan Yu, Jun Guo* and Yan Kong*

\begin{abstract}
Background: Melanoma is one of the most aggressive cancers with extremely poor prognosis, and the median survival time for stage IV patients is approximately 6 to 8 months. Unlike cutaneous melanoma, mucosal melanoma is a rare melanoma subtype among Caucasian patients but its incidence remains as high as $22.6 \%$ among Chinese patients. Screening specific genetic variations is the guideline to select targeted drugs for the treatment of advanced melanoma, whereas the genetic variation spectrum and potential therapeutic targets for mucosal melanoma are largely unclear. It is urgent to identify promising genetic variants for mucosal melanoma so as to develop effective targeted therapies for this disease.

Methods: Tumor samples from 213 Chinese mucosal melanoma patients were involved in this study. P16 $6^{\text {INK4a }} /$ Cyclin D1/CDK4 copy number was examined using the QuantiGene Plex DNA assay and the correlation between abnormal copy number and clinicopathological parameters was analyzed. Patient-derived xenograft models (PDX) were performed to detect the effects of CDK4/6 inhibitors on the proliferation of mucosal melanoma cells with altered copy number of CDK4 pathway (CDK4, Cyclin D1 and P16 $\left.6^{\text {IKKa }}\right)$. The molecular mechanisms of CDK4/6 inhibitors on the proliferation of mucosal melanoma were analyzed by RNAseq.
\end{abstract}

Results: Among the 213 samples, the amplification rate of CDK4 and CCND1 was $47.0 \%$ and $27.7 \%$, respectively, and the deletion rate of $P 16^{I N K 4 a}$ was $57.7 \%$. Patients with more than one genetic abnormality were up to $81.7 \%$. CDK4 pathway gene copy number variation was not associated with the prognosis of patients with mucosal melanoma $(P>0.05)$. Drug sensitivity tests showed that AT7519, a broad-spectrum CDK inhibitor, and PD0332991, a specific CDK4/6 inhibitor, exhibited higher inhibitory effect on CDK4 signaling pathway abnormal mucosal melanoma cellsderived PDX tumors growth than CDK4 signaling pathway normal ones. RNA-seq analysis showed that CDK4 inhibitors may affect tumor proliferation through multiple signaling pathways.

Conclusions: Abnormal copy number of cell cycle related genes is frequently found in mucosal melanoma. CDK4/6 inhibitors significantly suppress the PDX tumor growth with abnormal CDK4 pathway. CDK4 signaling variations predict the effectiveness of CDK4 inhibitors in mucosal melanoma.

Keywords: Mucosal melanoma, Targeted therapy, CDK4, CCND1, P16 $6^{\text {INK4a }}$

*Correspondence: guoj307@126.com; k-yanbjcancer@163.com

Key Laboratory of Carcinogenesis and Translational Research (Ministry

of Education), Department of Renal Cancer and Melanoma, Peking

University Cancer Hospital \& Institute, 52 Fucheng Road, Beijing 100142,

China

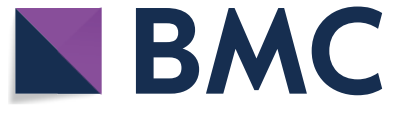

(c) The Author(s) 2019. This article is distributed under the terms of the Creative Commons Attribution 4.0 International License (http://creativecommons.org/licenses/by/4.0/), which permits unrestricted use, distribution, and reproduction in any medium, provided you give appropriate credit to the original author(s) and the source, provide a link to the Creative Commons license, and indicate if changes were made. The Creative Commons Public Domain Dedication waiver (http://creativecommons.org/ publicdomain/zero/1.0/) applies to the data made available in this article, unless otherwise stated. 


\section{Background}

Melanoma is one of the most aggressive cancers with extremely poor prognosis. Approximately 100,000 new cases of cutaneous melanoma (CM) are diagnosed and 7000 death occurs in the US in 2019 [1]. In China, the incidence of malignant melanoma is gradually growing, with an average of more than 20,000 new cases [2]. Because of the increased number of melanoma patients, more attention has been paid to the prevention and treatment of melanoma in China. Mucosal melanoma (MM), an aggressive subtype of melanoma, is extremely rare among Caucasian melanoma patients, with the incidence rate less than $2 \%$ [3]. However, the incidence of $M M$ is very high, accounting for $22.6 \%$ among Chinese melanoma patients [4]. Compared with other subtypes, MM is characterized by occult location, late stage of initial diagnosis, high recurrence and metastasis rate. Due to the difficulty of diagnosis and treatment, the prognosis of MM is significantly poor, with a $14 \% 5$-year survival. For Stage IV patients, the average survival time was only 6-8 months, and the 5-year survival rate was less than $5 \%[4,5]$. It is important to develop promising diagnostic biomarkers and effective treatment strategies for MM.

Genomic analysis shows that the major genetic variation of $\mathrm{MM}$ is the large-scale structural amplification or deletion of chromatin, distinct from the point mutation hotspot in CM [6-9]. Therefore, MM is different from $\mathrm{CM}$ in its pathogenesis, diagnosis, treatment and response to drugs. For instance, BRAF active mutation is common $(>50 \%)$ in $\mathrm{CM}$, whereas the mutation rate is only about $10 \%$ in MM $[6,10]$. Moreover, the effect of such targeted therapy on the treatment for MM was unclear due to the limited numbers of $\mathrm{MM}$ patients included in clinical trials. Copy number aberrations study may be helpful to investigate the pathogenesis of MM and to screen effective drug targets.

Abnormal cell cycle progression, caused by the mutation or amplification of CDK or cell cycle families, is one of the hallmarks of tumor cells [11]. CDKN2A/CDK4/6/ CCND1 complex is the essential regulator of cell cycle. CDK4/6/CCND1 complex phosphorylates the retinoblastoma $(\mathrm{Rb})$ and inhibits its activity, leading to the transition of G1 to S phase. By contrast, CDKN2A negatively regulates the progression of cell cycle. The combination of Palbociclib (a specific inhibitor of CDK4/6) and MLN0128 (an inhibitor of mTOR) significantly inhibits the proliferation of patient derived xenograft (PDX) model of ER-positive breast cancer [12]. High throughput sequencing in nasopharyngeal carcinoma reveals an increase in CCND1 copy number combined with CDKN2A gene deletion. Palbociclib significantly inhibits CDK4 signaling pathway activation in patient derived xenograft (PDX) model [13]. Palbociclib has antitumor activity for NRAS-mutant melanomas in a preclinical mouse model, indicating the CDK4 pathway as a potential therapeutic target [14]. A large cohort analysis in 2017 found that CDK4 gene amplification was higher in acral melanoma (AM), suggesting that CDK4 may be a therapeutic target for AM [15-17].

It is not clear whether there are variations in cell cycle related signaling pathways in $\mathrm{MM}$ and whether they can be used as effective therapeutic targets. To address this question, we collected $213 \mathrm{MM}$ samples and investigated the status of CDK4 signaling related genes (CDK4, CCND1 and CDKN2A) and its relationship with clinical pathologic parameters. We also explored the inhibitory effects of CDK4/6 inhibitors on PDX tumor growth containing normal or aberrant CDK4 pathway. The results of this study may provide a new idea for the clinical treatment of MM.

\section{Materials and methods}

\section{Patients and tissue samples}

This study involved samples from primary lesions of 213 MM patients, who were hospitalized during January 2007 and October 2018 at the Peking Cancer Hospital \& Institute. We obtained all the clinical and pathological data by medical record review, including age, gender, ulceration, depth of invasion, anatomic site, treatment, tumor-nodemetastases stage, mutation status of therapeutic targets (such as KIT, BRAF and NRAS), follow-up time and survival (follow-up persisted until June 2018, or until the missing of follow-up or death of patients). An informed consent was obtained from all participants that were enrolled in clinical trials. This study was approved by the Medical Ethics Committee of the Beijing Cancer Hospital \& Institute and was conducted according to the Declaration of Helsinki Principles.

\section{QuantiGenePlex DNA assay}

Tissue homogenates were prepared according to the protocols in the user manual of QuantiGene Sample Processing Kit for Formalin-Fixed, Paraffin-Embedded Tissues (FFPE; Panomics of Affymetrics, Santa Clara, CA). Briefly, 5 to 8 pieces of deparaffinized sections (4 to $10 \mu \mathrm{m}$ ) were incubated with $150 \mu \mathrm{l}$ homogenizing solution supplemented with $1.5 \mu \mathrm{l}$ of proteinase $\mathrm{K}(50 \mu \mathrm{g} / \mu \mathrm{l})$ at $65^{\circ} \mathrm{C}$ for $6 \mathrm{~h}$. The tissue homogenates were separated from debris by brief centrifugation and transferred to a new tube.

The branched DNA (bDNA) assay was performed according to the procedures described in the user manual of QuantiGenePlex DNA Assay (Panomics). Briefly, the homogenate DNA was sheared by the Covaris S2 (Covaris, Woburn, MA) with the following settings: duty cycle $5 \%$, intensity 3 , cycles/burst $200,80 \mathrm{~s}$. For each assay well, 
$40 \mu \mathrm{l}$ homogenate was denatured with $2.5 \mathrm{M} \mathrm{NaOH}$ (final concentration $0.18 \mathrm{M}$ ) in the presence of DNA probe. Neutralized tissue homogenate was transferred to each well of the Hybridization Plate containing Working Bead Mix. All the samples were in duplicates. Hybridization Plate was sealed and incubated at $54{ }^{\circ} \mathrm{C} \pm 1{ }^{\circ} \mathrm{C}$ in a shaking incubator $(600 \mathrm{rpm}$ ) for $18-22 \mathrm{~h}$. The unbound samples were washed away using the Bio-plex pro II wash station (Bio-Rad, Hercules, CA). Then the beads were sequentially hybridized with the DNA Pre-Amplifier, DNA Amplifier, Label Probe and SAPE (Streptavidinconjugated R-phycoerythrin). Fluorescence intensities were measured by the Bio-plex 100 system (Bio-Rad).

The mean fluorescence intensities of the duplicates were calculated for all genes. The background values were subtracted from each probe set signal. Values of tested genes were normalized to the geometric means of Rpph1, Rpp30 and Rplp0. For each test sample, normalized signal was divided by the reference DNA sample (G1521, Promega, Madison, WI) for each test gene, and the values were multiplied by the known copy number (usually 2 copies) of each gene in the reference genome. The $C d k 4, C c n d 1$ and $P 16^{I N K 4 a}$ copy numbers for the samples were calculated by dividing the sample values tothe control values: No gain referred to copy number $\leq 2$, gain referred to copy number $>2$, loss referred to copy number $<1$.

\section{DNA preparation and TaqMan copy number assays}

Genomic DNA was extracted from FFPE sections using a QIAamp DNA FFPE Tissue Kit (Qiagen, Hilden, Germany). To validate the results of QuantiGenePlex DNA Assay, the copy numbers of Cdk4, Ccnd1 and P16 $6^{\mathrm{INK} 4 \mathrm{a}}$ were further quantified by TaqMan Copy Number Assays (Applied Biosystems of ThermoFisher, Waltham, MA). A TaqMan probe targeted on the Rnasep gene was used as a control. Quantitative real-time PCR was performed using the ABI 7500 FAST real-time PCR system (Applied Biosystems). Copy numbers were then determined by CopyCaller v2.0 software (Applied Biosystems) using the comparative $\mathrm{Ct}(\Delta \Delta \mathrm{Ct})$ method.

\section{Immunohistochemistry assay}

Immunohistochemistry analyses were performed using antibodies against Ki67 (dilution 1:400) (Abcam, Cambridge, UK) as described $(11,17)$. The Ki- $67+$ cells under 5 random fields were counted and were presented as mean \pm SD of three sections.

\section{Cell culture}

The HMVII cell line was obtained from Sigma-Aldirch and was cultured at $37{ }^{\circ} \mathrm{C}$ in Ham's F-10 medium supplemented with $1 \%$ penicillin and streptomycin
(Invitrogen) and $10 \%$ fetal bovine serum (HyClone of GE Healthcare, Logan, UT). The GAK cell line was obtained from JCRB Cell Bank and was cultured at $37{ }^{\circ} \mathrm{C}$ in Ham's F-12 medium supplemented with $1 \%$ penicillin and streptomycin (Invitrogen) and 10\% fetal bovine serum (HyClone of GE Healthcare, Logan, UT).

\section{Cell proliferation assays}

CDK4/6 inhibitors including PD0332991 (\#S1116), and pan-CDK inhibitor AT7519 (\#S1524) were purchased from Selleck Chemicals (Houston, TX). All inhibitors were dissolved at $10 \mathrm{mM}$ in dimethylsulfoxide (DMSO) as stock solutions. After treatment with various concentrations of inhibitors or DMSO for $24 \mathrm{~h}$, proliferation of the cells was evaluated using the Cell Titer-Glo Luminescent Cell Viability Assay (Promega) according to the instructions.

\section{Patient-derived xenograft (PDX) model and treatment}

Fragments of patient-derived MM tissues bearing typical CDK4 pathway aberrations were cut into fragments and then subcutaneously inoculated into a 6 week-old NOD/SCID (non-obese diabetic and severe combined immunodeficiency) female mouse (4-6 week-old, 18-22 g-weight) to establish the PDX model. The established PDX model was called passage 0 (P0). When the tumor size reached approximately $500 \mathrm{~mm}^{3}$, the mice were sacrificed, and tumor tissues were separated and re-inoculated into new mice to obtain the subsequent passages called P1, P2, P3 and forth. 10 PDX models containing typical CDK4 pathway aberrations (Table 1) were finally established.

Mice (P2) were randomized (treatment arm versus control arm; $\mathrm{n}=4$ ) and treated with control (sodium lactate buffer, $\mathrm{pH}$ 4.5) or PD0332991 and with control (saline solution, pH 4.0) or AT7519. For PD0332991 treatment, mice received PD0332991 (50 mg/kg in pH 4.5 sodium lactate buffer) via oral gavage daily. For AT7519 treatment, mice received AT7519 (12 mg/ $\mathrm{kg}$ in saline solution) via intraperitoneal injection daily. Tumor sizes were measured every 3 days and tumor volume was calculated using the formula: volume $=$ length ${ }^{*}$ width $^{2} / 2$. Percentage of tumor volume on day of treatment (baseline volume) was used as the end-point of study. The treatment lasted for 14 days, after which the mice were sacrificed and the tumors were fixed in $10 \%$ formalin for histological and immunohistological analysis. All animal care and experimental procedures were performed in consistent with the Animal Care Ethics approved by the Medical Ethics Committee of the Beijing Cancer Hospital \& Institute. 
Table 1 The basic information of 10 PDX models

\begin{tabular}{|c|c|c|c|c|c|c|c|c|c|c|c|}
\hline Code & Gender & Age & Ulceration & $\begin{array}{l}\text { Anatomic } \\
\text { site }\end{array}$ & Stage & $C D K 4$ & CCND1 & $P 16^{I N K 4 a}$ & $K I T$ & $B R A F$ & NRAS \\
\hline PDX-001 & $M$ & 49 & Yes & 1 & IV & Normal & Normal & Normal & WT & WT & WT \\
\hline PDX-002 & $M$ & 57 & Yes & 4 & IV & Normal & Normal & Normal & W557R & WT & WT \\
\hline PDX-003 & $\mathrm{F}$ & 78 & Yes & 5 & $\|$ & Gain & Gain & Loss & WT & WT & WT \\
\hline PDX-004 & $\mathrm{F}$ & 43 & Yes & 5 & III & Gain & Gain & Loss & L576P & WT & WT \\
\hline PDX-005 & $\mathrm{F}$ & 57 & Yes & 5 & $\|$ & Gain & Gain & Loss & WT & WT & $\mathrm{WT}$ \\
\hline PDX-006 & $M$ & 58 & Yes & 3 & 1 & Gain & Gain & Loss & WT & WT & WT \\
\hline PDX-007 & $\mathrm{F}$ & 71 & Yes & 2 & $\|$ & Gain & Gain & Normal & WT & WT & WT \\
\hline PDX-008 & $\mathrm{F}$ & 65 & No & 1 & $\|$ & Gain & Gain & Normal & WT & WT & G12C \\
\hline PDX-009 & $\mathrm{F}$ & 69 & Yes & 1 & $\|$ & Normal & Gain & Loss & WT & WT & Q61L \\
\hline PDX-010 & $M$ & 55 & Yes & 2 & $\|$ & Normal & Gain & Normal & WT & WT & WT \\
\hline
\end{tabular}

$F$ female, $M$ male, $W T$ wild type

a: Anatomic site: $1=$ nasopharynx; 2 =oral cavities; $3=$ esophagus; $4=$ anorectum; $5=$ genitourinary

\section{RNA-sequencing}

RNA-sequencing was performed at the Shanghai Biotechnology Company. cDNA library was built according to the standard manufacturer's protocol. Paired-end $2 \times 100 \mathrm{bp}$ read sequencing was performed using the Illumina HiSeq X-ten (Illumina, USA). The FASTXToolkit (v0.0.13) was used to trim low-quality bases. High-quality reads were aligned to the human GRCh38 reference genome with two mismatches using spliced mapping alignment in Hisat2 (version: 2.0.4). After genome mapping, Stringtie (version: 1.3.0) was run with a reference annotation to generate FPKM values for known gene models. Differentially expressed genes were identified using edgeR. The $P$ value significance threshold in multiple tests was set by the false discovery rate (FDR). The fold-changes were also estimated according to the FPKM (Fragments Per Kilobase of exon model per Million mapped read) in each sample. The differentially expressed genes were selected using the following filter criteria: $F D R \leq 0.05$ and fold-change $\geq 2$.

\section{Statistical analysis}

Statistical analyses were performed using SPSS 22.0 software. Continuous data such as age was described using mean $\pm S D$ for normally distributed data. The correlations between aberration status and clinical parameters were evaluated by Chi square test or Fisher's exact test. Kaplan-Meier estimates of time-toevent overall survival (OS) and follow-up time were calculated. Log-rank tests were used to estimate the statistical significance between the time-dependent outcomes of OS. All statistical analyses were two-sided, and $P<0.05$ was considered as statistically significant.

\section{Results}

Aberrations of Cdk4, Ccnd1 and P16 ${ }^{\text {INK4a }}$ in MM

Firstly, we detected the copy number variation of CDK4, CCND1 and P16 ${ }^{\mathrm{INK} 4 \mathrm{a}}$ genes in paraffin sections of 213 cases of MMs through QGP method (Table 2). Among the 213 samples, 100 cases (47.0\%), 59 cases $(27.7 \%)$ and 123 cases $(57.7 \%)$ showed Cdk4 gain, Ccnd1 gain, and P16 ${ }^{\mathrm{INK} 4 a}$ loss, respectively. Moreover, $36.6 \%$ of $\mathrm{MMs}$ contained more than two concurrent aberrations, and $8.0 \%$ of MMs contained three aberrations. The overall frequency of $\mathrm{MM}$ containing any $\mathrm{CNV}(\geq 1 \mathrm{CNV})$ was $81.7 \%$, whereas 39 cases harbored no CNV aberrations in these three genes. 38 cases had Ccnd1 loss, and one case harbored P16 ${ }^{\mathrm{INK} 4 \mathrm{a}}$ gain (Table 2). Collectively, Cdk4 gain, Ccnd1 gain and/or P16 ${ }^{\mathrm{INK} 4 \mathrm{a}}$ loss were observed in most MMs.

\section{Correlation of CDK4 pathway aberrations to other driver genes}

We also detected the mutation status of c-Kit (exon 9, 11, 13, 17 and 18), BRAF (exon 11 and 15) and NRAS (exon 1 and 2) by Sanger sequencing. The result showed that the mutation rate of c-Kit, BRAF and NRAS was $7.4 \%$, $5.2 \%$ and $15.7 \%$ in $\mathrm{MM}$ patients carrying $\mathrm{Cdk} 4$ gain, respectively. For MM patients carrying Ccnd1 gain, the mutation rate of $\mathrm{c}-\mathrm{Kit}$, BRAF and NRAS was $1.8 \%, 8.9 \%$, $10.4 \%$, respectively. For MM patients carrying $\mathrm{P} 16^{\mathrm{INK} 4 \mathrm{a}}$, the mutation rate of c-Kit, BRAF and NRAS was 9.6\%, $4.3 \%, 10.5 \%$, respectively (Table 2 ).

\section{Correlation of Anatomic site to TNM stage of mucosal melanoma}

We next explored the anatomic sites of MM in our 213 MM patient's cohort. The prevalent anatomic sites were head and neck (44.2\%), anorectum (21.1\%), genitourinary 
Table 2 Copy number variations of genes related to CDK4 pathway and mutation status of therapeutic targets in mucosal melanoma

\begin{tabular}{|c|c|c|c|c|}
\hline \multirow[t]{3}{*}{ CNV status } & \multirow{3}{*}{$\begin{array}{l}N=213 \\
N(\%)\end{array}$} & \multicolumn{3}{|c|}{ Genetic mutation of therapeutic targets } \\
\hline & & \multicolumn{3}{|c|}{$\%$ (No. positive cases/no. examined cases) } \\
\hline & & KIT & BRAF & NRAS \\
\hline \multicolumn{5}{|l|}{$\geq 1 \mathrm{CNV}$} \\
\hline CDK4 gain & $100(47.0)$ & $7.4(7 / 95)$ & $5.2(5 / 96)$ & $15.7(11 / 70)$ \\
\hline $2.5-5$ copies & $76(35.7)$ & $5.6(4 / 71)$ & $5.6(4 / 72)$ & $19.6(11 / 56)$ \\
\hline 5-10 copies & $14(6.6)$ & $11.8(2 / 14)$ & $0(0 / 14)$ & $0(0 / 10)$ \\
\hline$>10$ copies & $10(4.7)$ & $10.0(1 / 10)$ & $10.0(1 / 10)$ & $0(0 / 4)$ \\
\hline CCND1 gain & $59(27.7)$ & $1.8(1 / 56)$ & $8.9(5 / 56)$ & $10.4(5 / 48)$ \\
\hline $2.5-5$ copies & $49(23.0)$ & $0(0 / 46)$ & $10.9(5 / 46)$ & $112.8(5 / 39)$ \\
\hline $5-10$ copies & $9(4.2)$ & $11.1(1 / 9)$ & $0(0 / 9)$ & $0(0 / 8)$ \\
\hline$>10$ copies & $1(0.5)$ & $0(0 / 1)$ & $0(0 / 1)$ & $0(0 / 1)$ \\
\hline P16 INK4a loss & $123(57.7)$ & $9.6(11 / 114)$ & $4.3(5 / 115)$ & $10.5(9 / 86)$ \\
\hline Overall & $174(81.7)$ & $7.0(11 / 199)$ & $10.0(10 / 200)$ & $13.7(21 / 153)$ \\
\hline \multicolumn{5}{|l|}{$\geq 2 \mathrm{CNVs}$} \\
\hline CDK4 gain plusCCND1 gain & $13(6.1)$ & $0(0 / 12)$ & $0(0 / 12)$ & $20.0(2 / 10)$ \\
\hline CDK4 gain plus P16 $6^{I N K 4 a}$ loss & $49(23.0)$ & $12.8(6 / 47)$ & $4.2(2 / 48)$ & $12.1(4 / 33)$ \\
\hline CCND1 gain plus P16 $6^{\mid N K 4 a}$ loss & $16(7.5)$ & $6.3(1 / 16)$ & $0(0 / 16)$ & $15.4(2 / 13)$ \\
\hline Overall & 78 (36.6) & $9.3(7 / 75)$ & $3.9(3 / 76)$ & $14.3(8 / 56)$ \\
\hline \multicolumn{5}{|l|}{3 CNVs } \\
\hline Overall & $17(8.0)$ & $0(0 / 16)$ & $12.5(2 / 16)$ & $0(0 / 14)$ \\
\hline
\end{tabular}

CNV copy number variation

(25.8\%) and oesophagus (8.9\%) (Table 4). The proportion of patients with TNM stage I, II, III, and IV diseases were $2.8 \%, 53.5 \%, 28.6 \%$ and $15.1 \%$, respectively. The percentages of patients with stage IV of MM from head and neck were significantly lesser than other anatomic sites $(\mathrm{P}<0.001)$.

\section{Correlation of CDK4 pathway aberrations to clinicopathological features}

Last, we investigated the correlations between the aberration of $\mathrm{Cdk} 4$, Ccnd 1 and $\mathrm{P} 16^{\mathrm{INK} 4 \mathrm{a}}$ and the clinicopathological features of MM. Statistical analysis was divided into 8 groups: Cdk4 aberration, Ccnd1 aberration, $\mathrm{P} 16^{\mathrm{INK} 4 \mathrm{a}}$ aberration, $\geq 1 \mathrm{CNV}$ (copy number variation), Cdk4 gain $+C$ cnd1gain, $C d k 4$ gain $+\mathrm{P} 16^{\mathrm{INK} 4 \mathrm{a}}$ loss, Ccnd1gain $+\mathrm{P} 16^{\mathrm{INK} 4 \mathrm{a}}$ loss and Cdk4 gain $+\mathrm{Ccnd}-$ 1gain + P16 ${ }^{\mathrm{INK} 4 \mathrm{a}}$ loss.

In our cohort, both age, gender, anatomic site and follow-up time were not significantly different between patients with or without any CNVs for Cdk4, Ccnd1, P16 ${ }^{\text {INK4a }}$ or other indicated stochastic combinations. However, the other clinical features were at least significantly associated with a molecular variant of the CDK4 signaling pathway: compared with $\mathrm{P} 16^{\mathrm{INK} 4 \mathrm{a}}$ loss, patients with normal were more likely to ulcerate; The depth of invasion of $\mathrm{P} 16^{\mathrm{INK} 4 \mathrm{a}}$ normal group tended to be deeper than that of group with $\mathrm{P} 16^{\mathrm{INK} 4 \mathrm{a}}$ loss, while there was no statistical difference $(\mathrm{P}=0.053)$. The percentages of patients with stage I-IV of MM were significantly different between patients with CDK4 gain and CDK4 normal $(\mathrm{P}=0.004$; Table 3$)$. The TNM stage of $\geq 1 \mathrm{CNV}$ group tended to be greater than that of group without any CDK4 pathway aberrations, while there was no statistical difference $(\mathrm{P}=0.059)$; Similar result was observed in the group of CDK4 gain $+\mathrm{P} 16^{\mathrm{INK} 4 \mathrm{a}}$ loss $(\mathrm{P}=0.088)$. The treatment groups for MM patients was significantly different between patients with CDK4 gain and CDK4 normal $(\mathrm{P}=0.003$; Additional file 1: Table S1).

The overall survival of MM patients with $\mathrm{Cdk} 4$ gain, Ccnd1 gain, P16 ${ }^{\mathrm{INK} 4 \mathrm{a}}$ loss or other indicated stochastic combinations were comparable. Univariate Cox analysis suggested thatCdk4 gain, Ccnd1 gain, P16 ${ }^{\mathrm{INK} 4 \mathrm{a}}$ loss or other stochastic combinations might not be of prognostic significance for melanoma patients (Table 3).

\section{Sensitivity of MM cells to CDK4/6 inhibitors}

To evaluate the effect of CDK4/6 inhibition on the proliferation of MM cells, GAK and HMV II cell lines were treated with different concentrations of panCDK inhibitor AT7519 and CDK4/6 specific inhibitor 
Table 3 Correlation of CDK4 pathway aberrations to clinicopathologic features of mucosal melanoma

\begin{tabular}{|c|c|c|c|c|c|c|c|}
\hline \multirow[t]{2}{*}{ Clinicopathologic factor } & \multicolumn{3}{|c|}{ CDK4 aberration } & \multicolumn{4}{|c|}{ CCND1 aberration } \\
\hline & Gain & Normal & $P$ value & Gain & Loss & Normal & $P$ value \\
\hline Age (year) & & & 0.552 & & & & 0.853 \\
\hline Median (range) & $56.5 \pm 11.6$ & $54.9 \pm 11.3$ & & $56.4 \pm 11.3$ & $55.3 \pm 11.6$ & $55.4 \pm 11.6$ & \\
\hline Gender n (\%) & & & 0.409 & & & & 0.413 \\
\hline Male & $41(41.0)$ & $39(35.5)$ & & $20(33.9)$ & $18(47.4)$ & 45 (38.8) & \\
\hline Female & $59(59.0)$ & $71(64.5)$ & & $39(66.1)$ & $20(52.6)$ & $71(61.2)$ & \\
\hline Ulceration n (\%) & & & 0.555 & & & & 0.769 \\
\hline Yes & $52(52.0)$ & $49(44.5)$ & & $25(42.4)$ & $20(52.6)$ & $58(50.0)$ & \\
\hline No & $20(20.0)$ & $26(23.6)$ & & $14(23.7)$ & $9(23.7)$ & $23(19.8)$ & \\
\hline NA & $28(28.0)$ & $35(31.8)$ & & $20(33.9)$ & $9(23.7)$ & $35(30.2)$ & \\
\hline Depth of invasion & & & 0.981 & & & & 0.853 \\
\hline $\mathrm{T} 1 \leq 1 \mathrm{~mm}$ & $13(13.0)$ & $16(14.5)$ & & $10(16.9)$ & $4(10.5)$ & 15 (12.9) & \\
\hline $\mathrm{T} 21-2 \mathrm{~mm}$ & $21(21.0)$ & $24(21.8)$ & & $10(16.9)$ & $11(28.9)$ & $25(21.6)$ & \\
\hline T3 2-4 mm & $18(18.0)$ & $20(18.2)$ & & $10(16.9)$ & $6(15.8)$ & $22(19.0)$ & \\
\hline $\mathrm{T} 4>4 \mathrm{~mm}$ & $48(48.0)$ & $50(45.5)$ & & $29(49.2)$ & $17(44.7)$ & $54(46.6)$ & \\
\hline Anatomic site n (\%) & & & 0.153 & & & & 0.885 \\
\hline Head and neck & $51(51.0)$ & $41(37.3)$ & & $27(45.8)$ & $15(39.5)$ & $52(44.8)$ & \\
\hline Oesophagus & $8(8.0)$ & $11(10.0)$ & & $3(5.1)$ & $5(13.2)$ & $11(9.5)$ & \\
\hline Anorectum & $21(21.0)$ & $23(20.9)$ & & $13(22.0)$ & $9(23.7)$ & $23(19.8)$ & \\
\hline Genitourinary & $20(20.0)$ & $35(31.8)$ & & $16(27.1)$ & $9(23.7)$ & $30(25.9)$ & \\
\hline Stages n (\%) & & & 0.004 & & & & 0.239 \\
\hline 1 & $5(5.0)$ & $1(0.9)$ & & $0(0.0)$ & $1(2.6)$ & $5(4.3)$ & \\
\hline$\|$ & $46(46.0)$ & $67(60.9)$ & & $30(50.8)$ & $26(68.4)$ & $58(50.0)$ & \\
\hline III & $38(38.0)$ & $22(20.0)$ & & $18(30.5)$ & $9(23.7)$ & $34(29.3)$ & \\
\hline IV & $11(11.0)$ & $20(18.0)$ & & $11(18.6)$ & $2(5.3)$ & $19(16.4)$ & \\
\hline Survival (months) & & & 0.054 & & & & 0.702 \\
\hline Median $(95 \% \mathrm{Cl})$ & $45.0(39.3,50.7)$ & $41.2(35.4,47.0)$ & & $43(40.2,45.8)$ & $47.4(38.9,55.9)$ & $45.0(40.0,50.0)$ & \\
\hline Median follow-up time ( $95 \% \mathrm{Cl})$ & $38.1(26.0,50.2)$ & $37.0(25.5,48.5)$ & 0.322 & $44.5(27.4,61.6)$ & $39.5(22.2,56.8)$ & $31.0(18.3,43.7)$ & 0.453 \\
\hline \multirow[t]{2}{*}{ Clinicopathologic factor } & \multicolumn{2}{|c|}{ P16 ${ }^{\text {INK4a }}$ aberration } & & & \multicolumn{2}{|c|}{ Overall aberration ( $\geq 1 \mathrm{CNV}$ ) } & \\
\hline & Loss & Normal & & $P$ value & & No & $P$ value \\
\hline Age (year) & & & & 0.733 & & & 0.581 \\
\hline Median (range) & $55.4 \pm 11.5$ & $56.2 \pm 11.4$ & & & \pm 11.4 & $56.3 \pm 12.0$ & \\
\hline Gender n (\%) & & & & 0.903 & & & 0.771 \\
\hline Male & $48(39.0)$ & $34(38.2)$ & & & 38.5) & $16(41.0)$ & \\
\hline Female & $75(61.0)$ & $55(61.8)$ & & & (61.5) & $23(59.0)$ & \\
\hline Ulceration n (\%) & & & & 0.014 & & & 0.189 \\
\hline Yes & $52(42.3)$ & $51(57.3)$ & & & 46.6) & $22(56.4)$ & \\
\hline No & $25(20.3)$ & $21(23.6)$ & & & 20.7) & $10(25.6)$ & \\
\hline NA & $46(37.4)$ & $17(119.1)$ & & & 20.7) & $7(17.9)$ & \\
\hline Depth of invasion & & & & 0.053 & & & 0.348 \\
\hline $\mathrm{T} 1 \leq 1 \mathrm{~mm}$ & $20(16.3)$ & $9(10.1)$ & & & 14.9) & $3(7.7)$ & \\
\hline T2 1-2 mm & $33(26.8)$ & $13(14.6)$ & & & 23.0) & $6(15.4)$ & \\
\hline T3 2-4 mm & $19(15.4)$ & $19(21.3)$ & & & 16.7) & $9(23.1)$ & \\
\hline $\mathrm{T} 4>4 \mathrm{~mm}$ & $51(41.5)$ & $48(53.9)$ & & & 45.4) & $21(53.8)$ & \\
\hline Anatomic site n (\%) & & & & 0.379 & & & 0.691 \\
\hline Head and neck & $55(44.7)$ & $39(43.8)$ & & & 45.4) & $15(38.5)$ & \\
\hline Oesophagus & $14(11.4)$ & $5(5.6)$ & & & & $3(7.7)$ & \\
\hline Anorectum & $22(17.9)$ & $22(24.7)$ & & & 19.5) & $11(28.2)$ & \\
\hline Genitourinary & $32(26.0)$ & $23(25.8)$ & & & 25.9) & $10(25.6)$ & \\
\hline
\end{tabular}


Table 3 (continued)

\begin{tabular}{|c|c|c|c|c|c|c|}
\hline \multirow[t]{2}{*}{ Clinicopathologic factor } & \multicolumn{3}{|c|}{ P16 ${ }^{\text {INK4a }}$ aberration } & \multicolumn{3}{|c|}{ Overall aberration ( $\geq 1 \mathrm{CNV}$ ) } \\
\hline & Loss & Normal & $P$ value & Yes & No & $P$ value \\
\hline \multicolumn{3}{|l|}{ Stages n (\%) } & 0.515 & & & \\
\hline I & $4(3.3)$ & $2(2.2)$ & & $5(2.9)$ & $1(2.6)$ & 0.059 \\
\hline$\|$ & $65(52.8)$ & $49(55.1)$ & & $90(51.7)$ & $24(61.5)$ & \\
\hline III & $39(31.7)$ & $22(24.7)$ & & $56(32.2)$ & $5(12.8)$ & \\
\hline IV & $15(12.2)$ & $16(18.0)$ & & $23(13.2)$ & $9(23.1)$ & \\
\hline \multicolumn{3}{|l|}{ Survival (months) } & 0.528 & & & 0.169 \\
\hline Median $(95 \% \mathrm{Cl})$ & $43.6(40.0,47.2)$ & $43.6(32.3,54.9)$ & & $44.0(40.4,47.6)$ & $43.6(18.6,68.6)$ & \\
\hline Median follow-up time (95\% Cl) & $39.7(29.7,49.7)$ & $33.7(22.3,45.1)$ & 0.137 & $38.1(31.7,44.5)$ & $21.9(17.8,26.0)$ & 0.206 \\
\hline \multirow[t]{2}{*}{ Clinicopathologic factor } & \multicolumn{3}{|c|}{ CDK4 gain + CCND1 gain } & \multicolumn{3}{|c|}{ CDK4 gain + P16 INK4a loss } \\
\hline & Positive & Negative & $P$ value & Positive & Negative & $P$ value \\
\hline \multicolumn{3}{|l|}{ Age (year) } & 0.866 & & & 0.722 \\
\hline Median (range) & $57.6 \pm 11.1$ & $55.4 \pm 11.5$ & & $56.2 \pm 11.1$ & $55.5 \pm 11.6$ & \\
\hline \multicolumn{3}{|l|}{ Gender n (\%) } & 0.817 & & & 0.382 \\
\hline Male & $12(40.0)$ & $68(37.8)$ & & $28(42.4)$ & $52(36.1)$ & \\
\hline Female & $18(60.0)$ & $112(62.2)$ & & $38(57.6)$ & $92(63.9)$ & \\
\hline \multicolumn{3}{|l|}{ Ulceration n (\%) } & 0.690 & & & 0.971 \\
\hline Yes & $13(43.3)$ & $88(48.9)$ & & $31(47.0)$ & $70(48.6)$ & \\
\hline No & $6(20.0)$ & $40(22.2)$ & & $15(22.7)$ & $31(21.5)$ & \\
\hline NA & $11(36.7)$ & $52(28.9)$ & & $20(30.3)$ & $43(29.9)$ & \\
\hline \multicolumn{3}{|l|}{ Depth of invasion } & 0.767 & & & 0.527 \\
\hline $\mathrm{T} 1 \leq 1 \mathrm{~mm}$ & $3(10.0)$ & $26(14.4)$ & & $8(12.1)$ & $21(14.6)$ & \\
\hline $\mathrm{T} 21-2 \mathrm{~mm}$ & $5(16.7)$ & $40(22.2)$ & & $18(27.3)$ & $27(18.8)$ & \\
\hline T3 2-4 mm & $6(20.0)$ & $32(17.8)$ & & $10(15.2)$ & $28(19.4)$ & \\
\hline $\mathrm{T} 4>4 \mathrm{~mm}$ & $16(53.3)$ & $82(45.6)$ & & $30(45.5)$ & $68(47.2)$ & \\
\hline \multicolumn{3}{|l|}{ Anatomic site n (\%) } & 0.160 & & & 0.935 \\
\hline Head and neck & $16(53.3)$ & $76(42.2)$ & & $31(47.0)$ & $61(42.4)$ & \\
\hline Oesophagus & $3(10.0)$ & $16(8.9)$ & & $6(9.1)$ & $13(9.0)$ & \\
\hline Anorectum & $8(26.7)$ & $36(20.0)$ & & $13(19.7)$ & $31(21.5)$ & \\
\hline Genitourinary & $3(10.0)$ & $52(28.9)$ & & $16(24.2)$ & $39(27.1)$ & \\
\hline \multicolumn{3}{|l|}{ Stages n (\%) } & 0.213 & & & 0.088 \\
\hline 1 & $0(0.0)$ & $6(3.3)$ & & $4(6.1)$ & $2(1.4)$ & \\
\hline ॥ & $12(40.0)$ & $101(56.1)$ & & $32(48.5)$ & $81(56.2)$ & \\
\hline III & $12(40.0)$ & $48(26.7)$ & & $23(34.8)$ & $37(25.7)$ & \\
\hline IV & $6(20.0)$ & $25(13.9)$ & & $7(10.6)$ & $24(16.7)$ & \\
\hline \multicolumn{3}{|l|}{ Survival (months) } & 0.757 & & & 0.174 \\
\hline Median (95\% Cl) & $44.0(41.4,46.6)$ & $43.6(39.3,47.9)$ & & $44.0(41.4,46.6)$ & $43.0(37.6,48.4)$ & \\
\hline Median follow-up time (95\% Cl) & $54.0(29.2,78.8)$ & $37.0(30.0,44.0)$ & 0.636 & $38.1(25.9,50.3)$ & $37.0(29.1,44.9)$ & 0.153 \\
\hline \multirow[t]{2}{*}{ Clinicopathologic factor } & \multicolumn{3}{|c|}{ P16 ${ }^{\text {INK4a }}$ loss + CCND1 gain } & \multicolumn{3}{|c|}{ CDK4 gain + P16 ${ }^{\text {INK4a }}$ loss + CCND1 gain } \\
\hline & Positive & Negative & $P$ value & Positive & Negative & $P$ value \\
\hline Age (year) & & & 0.531 & & & 0.807 \\
\hline Median (range) & $58.1 \pm 12.5$ & $55.3 \pm 11.2$ & & $59.2 \pm 12.8$ & $55.3 \pm 11.4$ & \\
\hline Gender n (\%) & & & 0.927 & & & 0.476 \\
\hline Male & $13(39.4)$ & $69(38.5)$ & & $8(47.1)$ & $75(38.3)$ & \\
\hline Female & $20(60.6)$ & $110(61.5)$ & & $9(52.9)$ & $121(61.7)$ & \\
\hline
\end{tabular}


Table 3 (continued)

\begin{tabular}{|c|c|c|c|c|c|c|}
\hline \multirow[t]{2}{*}{ Clinicopathologic factor } & \multicolumn{3}{|c|}{ P16 ${ }^{\text {INK4a }}$ loss + CCND1 gain } & \multicolumn{3}{|c|}{$\mathrm{CDK} 4$ gain $+\mathrm{P} 16^{\mathrm{INK} 4 \mathrm{a}}$ loss $+\mathrm{CCND} 1$ gain } \\
\hline & Positive & Negative & $P$ value & Positive & Negative & $P$ value \\
\hline Ulceration n (\%) & & & 0.220 & & & 0.857 \\
\hline Yes & $13(39.4)$ & $90(50.3)$ & & $8(47.1)$ & $95(48.5)$ & \\
\hline No & $6(18.2)$ & $40(22.3)$ & & $3(17.6)$ & $43(21.9)$ & \\
\hline NA & $14(42.4)$ & $49(27.4)$ & & $6(35.3)$ & $58(29.6)$ & \\
\hline Depth of invasion & & & 0.584 & & & 0.682 \\
\hline $\mathrm{T} 1 \leq 1 \mathrm{~mm}$ & $7(21.2)$ & $22(12.3)$ & & $1(5.9)$ & $28(14.3)$ & \\
\hline $\mathrm{T} 21-2 \mathrm{~mm}$ & $7(21.1)$ & $39(21.8)$ & & $3(17.6)$ & $43(21.9)$ & \\
\hline T3 $2-4 \mathrm{~mm}$ & $5(15.2)$ & $33(18.4)$ & & $3(17.6)$ & $35(17.9)$ & \\
\hline $\mathrm{T} 4>4 \mathrm{~mm}$ & $14(42.4)$ & $85(47.5)$ & & $10(58.8)$ & $90(45.9)$ & \\
\hline Anatomic site n (\%) & & & 0.404 & & & 0.497 \\
\hline Head and neck & $18(54.5)$ & $76(42.5)$ & & $9(52.9 .6)$ & $85(43.4)$ & \\
\hline Oesophagus & $1(3.0)$ & $18(10.0)$ & & $1(5.9)$ & $18(9.2)$ & \\
\hline Anorectum & $6(18.2)$ & $38(21.1)$ & & $5(29.4)$ & $40(20.4)$ & \\
\hline Genitourinary & $8(24.2)$ & $47(26.1)$ & & $2(11.8)$ & $53(27.0)$ & \\
\hline Stages n (\%) & & & 0.383 & & & 0.268 \\
\hline । & $0(0.0)$ & $6(3.4)$ & & $0(0.0)$ & $6(3.1)$ & \\
\hline$\|$ & $16(48.5)$ & $98(54.7)$ & & $6(35.3)$ & $108(55.1)$ & \\
\hline III & $13(39.4)$ & $48(26.8)$ & & $8(47.1)$ & $53(27.0)$ & \\
\hline IV & $4(12.1)$ & $27(15.1)$ & & $3(17.6)$ & $29(14.8)$ & \\
\hline Survival (months) & & & 0.287 & & & 0.145 \\
\hline Median (95\% Cl) & $42(35.0,49.0)$ & $45.5(40.8,50.2)$ & & $42(33.5,50.5)$ & $45(40.5,49.4)$ & \\
\hline Median follow-up time (95\% Cl) & $55.5(20.3,90.7)$ & $35.0(27.6,42.4)$ & 0.102 & $38.1(25.9,50.3)$ & $37.0(29.1,44.9)$ & 0.153 \\
\hline
\end{tabular}

Table 4 Correlation of Anatomic sites to TNM stage of mucosal melanoma

\begin{tabular}{|c|c|c|c|c|c|c|}
\hline \multirow[t]{2}{*}{ Anatomic site } & \multirow[t]{2}{*}{ Patients (no. \%) } & \multicolumn{4}{|c|}{ TNM分期 (no.\%) } & \multirow{2}{*}{$\begin{array}{l}\text { P-value } \\
<0.001\end{array}$} \\
\hline & & 1 & II & III & IV & \\
\hline Head and neck & $94(44.2)$ & $3(3.2)$ & $57(60.6)$ & $31(33.0)$ & $3(3.2)$ & \\
\hline Nasopharynx & $60(28.2)$ & $1(1.7)$ & $48(80.0)$ & $10(16.7)$ & $1(1.7)$ & \\
\hline Oral cavity & $34(16.0)$ & $2(5.9)$ & $9(26.5)$ & $21(61.8)$ & $2(5.9)$ & \\
\hline Oesophagus & $19(8.9)$ & $1(5.3)$ & $6(31.6)$ & $8(42.1)$ & $4(21.1)$ & \\
\hline Anorectum & $45(21.1)$ & $1(2.2)$ & $15(33.3)$ & $14(31.1)$ & $15(33.3)$ & \\
\hline Genitourinary $^{a}$ & $55(25.8)$ & $1(1.8)$ & $36(65.5)$ & $8(14.5)$ & $10(18.2)$ & \\
\hline Total & 213 & $6(2.8)$ & $114(53.5)$ & $61(28.6)$ & $32(15.1)$ & \\
\hline
\end{tabular}

a The sites of genitourinary mucosal melanomas included vulva, vagina, urethra and cervix

PD0332991. The genetic variations of key genes for these cells are listed in Table 4. Both pan-CDK inhibitor AT7519 and CDK4/6 specific inhibitor PD0332991 significantly inhibited the viability of GAK and HMV II cells. For AT7519, the inhibitory rate in GAK and HMV II cells was obvious when higher than $4 \mu \mathrm{mol} / \mathrm{l}$. For PD0332991, GAK and HMV II were strikingly sensitive at a concentration higher than $10 \mu \mathrm{mol} / \mathrm{l}$ (Fig. 1).
Sensitivity of PDX models to CDK4/6 inhibitors

To analyze the sensitivity of MM containing typical CDK4 pathway aberrations to CDK4/6 inhibitors, we selected 10 PDX models with 5 different CDK4 pathway aberrations. The basic information of the PDX models is shown in Table 1. Two models (PDX-001 and PDX002) are CDK4 pathway normal. Four models (PDX-003, PDX-004, PDX-005 and PDX-006) are Cdk4 gain $+C c n d 1$ 
a

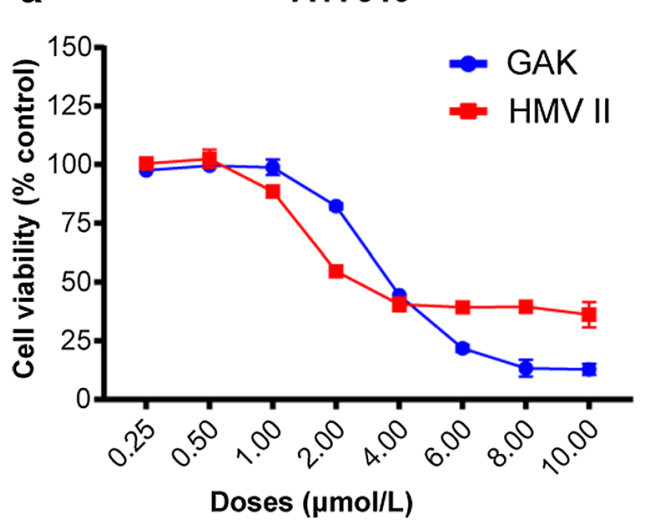

b

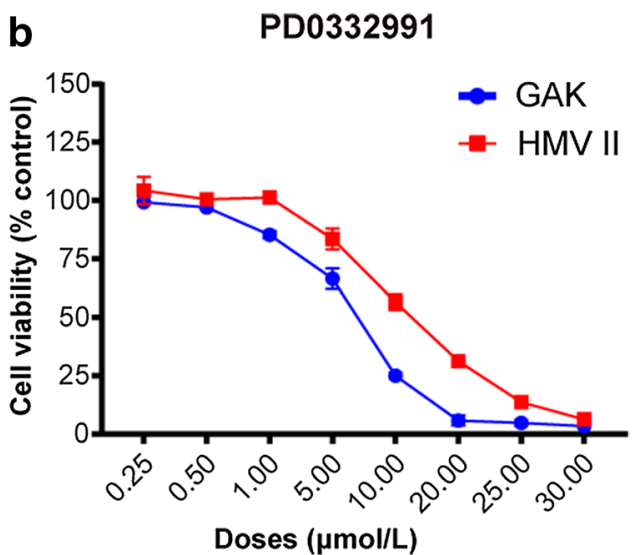

Fig. 1 Sensitivity of mucosal melanoma cells to CDK4/6 inhibitors. The proliferation was evaluated by Cell Titer-Glo method (a, b), and the results were presented as mean $\pm S D$ of three independent experiments. The statistical significance of the growth curves was evaluated by repeated measure variance analysis

gain $+P 16^{I N K 4 a}$ loss. Two models (PDX-007 and PDX008) are $C d k 4$ gain $+C c n d 1$ gain. One model (PDX-009) is Ccnd1 gain $+P 16^{I N K 4 a}$ loss. One model (PDX-010) is Ccnd1 gain.

Then we treated the PDX models with AT7519 and PD0332991. As compared to the vehicle-treated group, AT7519 and PD0332991 showed no inhibitory effect on tumor growth in PDX-001 and PDX-002 model. Interestingly, AT7519 and PD0332991 significantly retarded the tumor growth of PDX-003 to PDX-010 (Fig. 2). To confirm our findings, we performed immunohistochemical staining of Ki-67 in these tumors. Consistently, the number of Ki-67+ cells was significantly decreased after AT7519 and PD0332991 treatments in PDX models with CDK4 pathway aberration (Fig. 3). Taken together, these data indicate that CDK4 aberration dictates the sensitivity of MM-derived PDX tumor growth to CDK inhibitors (Table 5).

\section{Gene expression profile of PDX005 tumor tissue to CDK4/6 inhibitors}

To identify the molecular alterations after CDK4/6 inhibitors treatment, mRNA sequencing was performed on PDX tumor cells isolated from three paired tissue samples. On average, 7.6 million reads (between 7.0 and 8.6 million per sample) were obtained using Illumina HiSeq 2500 platform of which $97 \%$ had high quality scores ( $\geq$ Q20). Compared with vehicle group, AT7519 treated PDX-005 tumors had 1345 DEGs (FC $\geq 2$, adjusted P-values $<0.05$ ), among which 725 genes were down-regulated and 620 genes were up-regulated. In PD0332991-treated tumors, a total of 919 DEGs (FC $\geq 2$, adjusted $\mathrm{P}$-values $<0.05$ ) were observed, among which 648 genes were down-regulated and 271 genes were upregulate. The volcanic map of down- and up-regulated genes was listed in Fig. 4.

Functional annotation analysis of all DEGs utilizing: Profiler software revealed "Ribosome" (58 genes, $\mathrm{P}<7.8 \mathrm{E}-24)$, "Oxidative phosphorylation" (32 genes, $\mathrm{P}<4.8 \mathrm{E}-08)$, "Focal adhesion" $(29$ genes, $\mathrm{P}<0.001)$ and "Antigen processing and presentation" (15 genes, $\mathrm{P}<0.002$ ) were the most enriched KEGG pathways for AT7519 vs vehicle. For PD0332991 vs vehicle group, "Cell cycle" (25 genes, P $<7.5 \mathrm{E}-9$ ), "DNA replication" (9 genes, P-values 3.1E-05), "Focal adhesion" (25 genes, $\mathrm{P}<5.6 \mathrm{E}-05)$ and "p53 signaling pathway" (11 genes, $\mathrm{P}<0.0004$ ) were the most enriched KEGG pathways. The top 10 pathways were showed in Fig. 5.

\section{Discussion}

Melanoma is the fifth most common cancer in the Caucasian population [1]. CM is the major subtype melanoma in Caucasian population. Epidemiological statistics showed more than 5000 new cases of MM in China every year, and the incidence of $\mathrm{MM}$ is increasing [2]. Unlike $\mathrm{CM}, \mathrm{MM}$ exhibits distinct biological and clinical features.

(See figure on next page.)

Fig. 2 Sensitivity of PDX models containing CDK4 aberrations to CDK4/6 inhibitors in vivo. When the tumor size reached approximately 600 mm ${ }^{3}$, mice ( $n=4$ per group) were treated with buffer control or inhibitors daily. Tumor volume was evaluated as $\%$ of the tumor volume on day 0 and presented as mean \pm SD. The comparison of the growth curves was done with the repeated measure variance analysis. $n s$ no significances; ${ }^{* *} \mathrm{P}<0.01 ;{ }^{* * * P}<0.001$ 


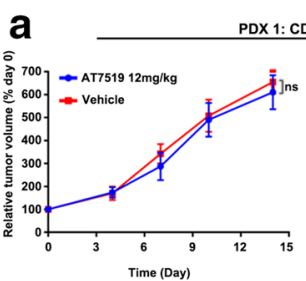

PDX 1: CDK4 Pathway Normal

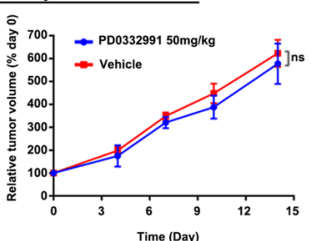

Control $\rightarrow \infty-\infty$

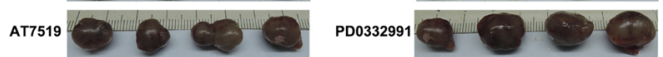
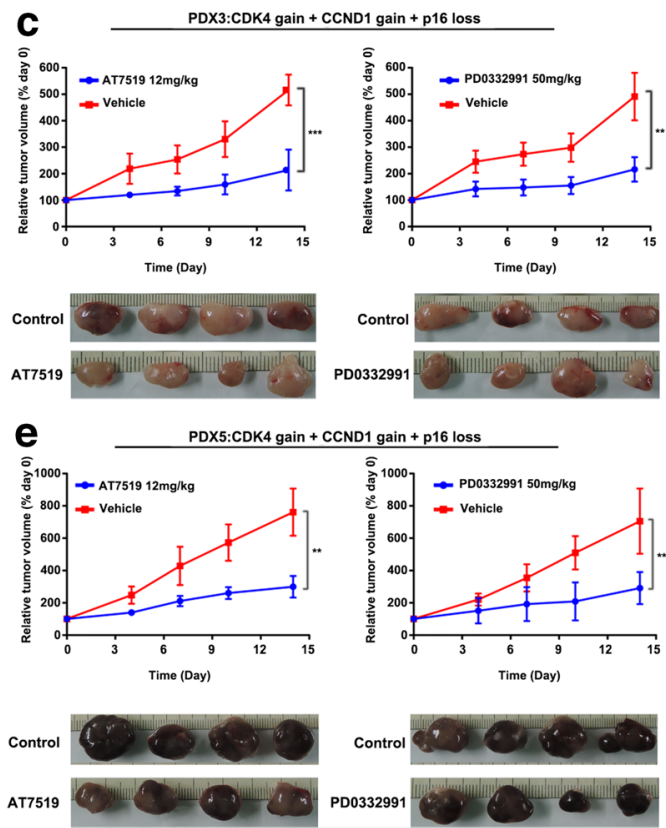

g

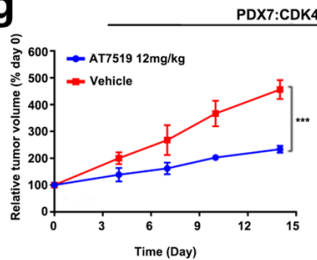

PDX7:CDK4 gain + CCND1 gain
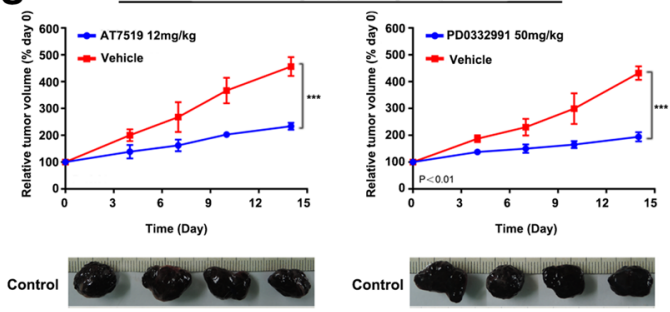

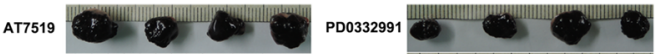
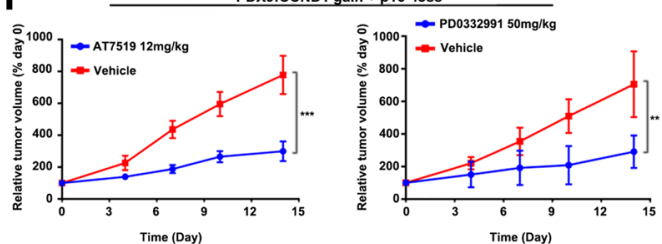

AT7519

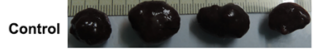

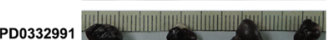
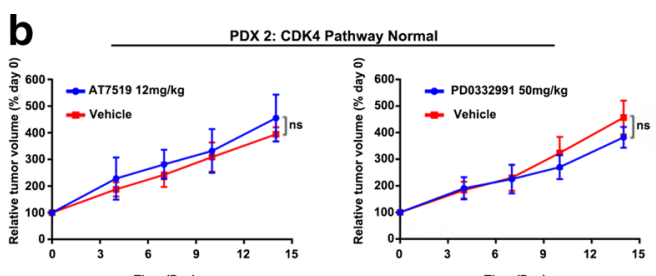

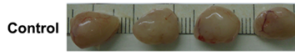

Control

Time (Day)

AT7519

PD0332991

d
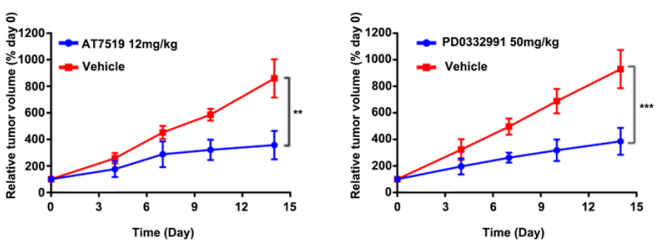

Control (2) (1)

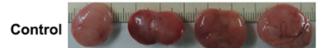

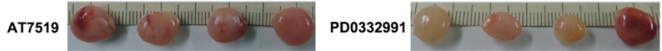

$f$
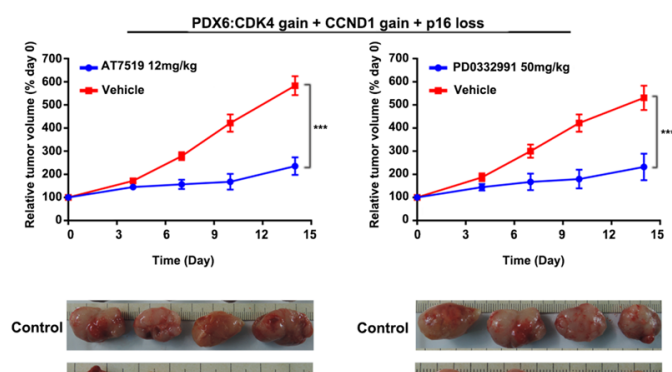

Control

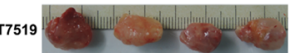

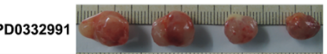

h
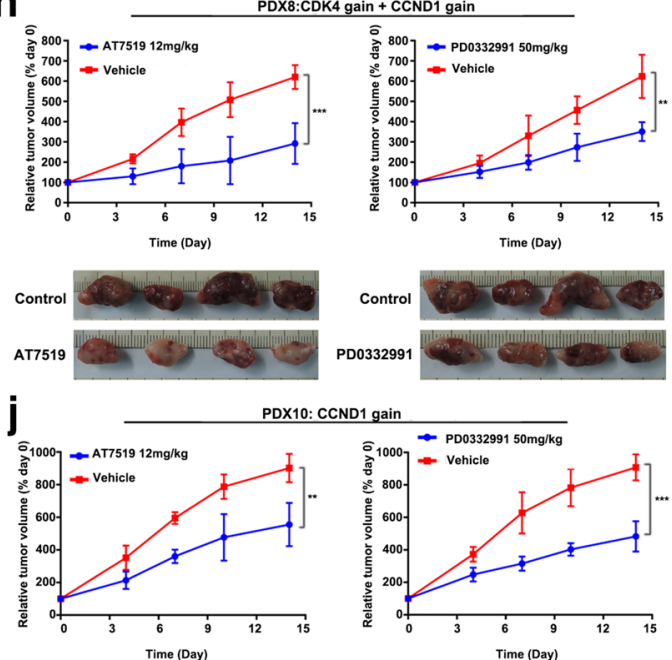

control 1000

control 1202

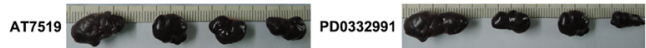




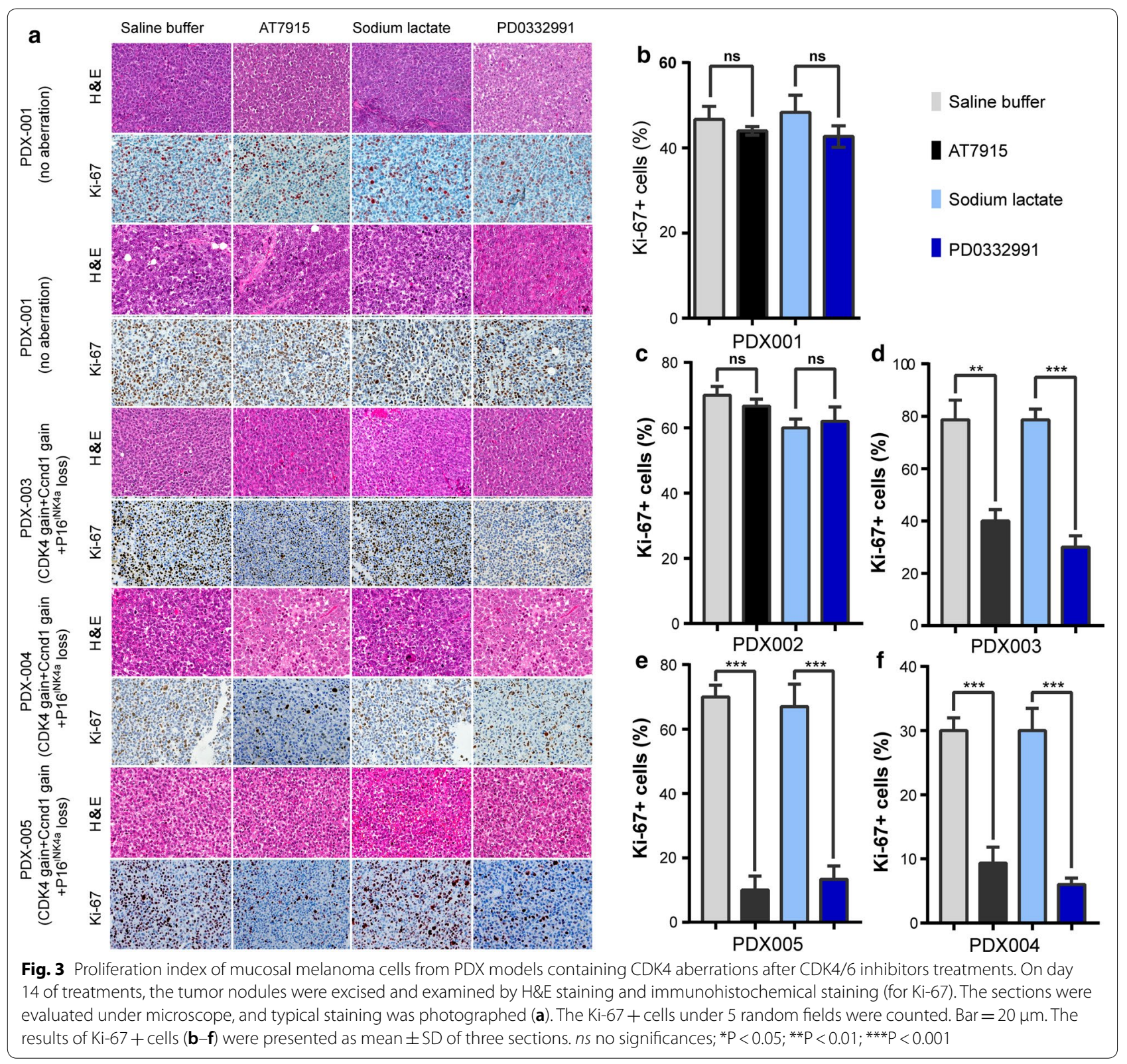

Targeted therapies, such as BRAF inhibitors and c-KIT inhibitors, have greatly revolutionized the treatment of CM. However, the effect of such targeted therapy on the treatment for MM remained obscure due to following reasons: 1 , the number of MM patients included in clinical trials is very limited. 2 , the mutation rates of the BRAF and c-KIT genes were only about 13.9 and 9.6\%, respectively, in MM patients $[18,19]$. Thus, potential targets should be identified for MM patients for developing new and effective targeted therapies.

Copy number variation of CDK signaling pathway genes plays an important role in many tumors. Reducing the activity of CDK signaling pathway can significantly inhibit the growth of tumor. CDK4/6 and CCND1 genes were amplified while CDKN2A, CDKN2B and CDKN1B were deleted in primary and metastatic breast cancers [20]. In patients with type 1 neurofibromatosis associated with breast cancer, the expansion of CDK4 copy number may increase the expression of the NF1 gene and then up-regulate the expression of Her2 gene in breast cancer cells [21]. CDK4 is amplified and its protein expression is increased in esophageal squamous cell carcinoma (ESCC), and both of them are associated with the poor survival of ESCC. CDK4 silencing or 


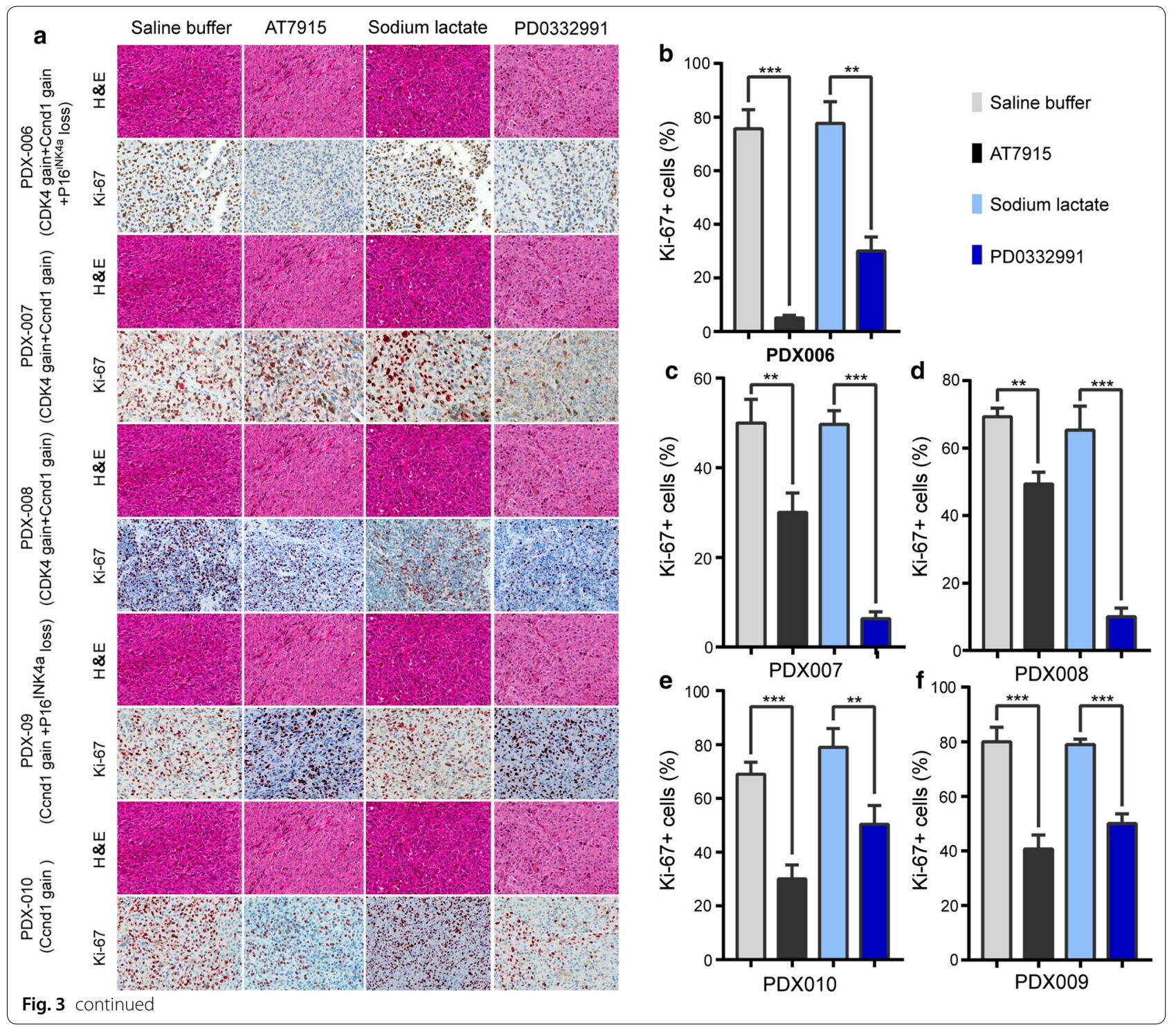

Table 5 The mutation status of GAK and HMV II

\begin{tabular}{lllllll}
\hline Cells & CDK4 & CCND1 & P16 INK4a & BRAF & NRAS $^{a}$ & CKIT $^{a}$ \\
\hline GAK & Gain & Gain & Normal & WT & Q61L & WT \\
HMV II & Gain & Normal & Loss & G469L & Q61K & WT \\
\hline
\end{tabular}

PD0332991 treatment significantly inhibits the proliferation of esophageal cancer cells [22]. Similar results are observed that CDK4/6 inhibitors suppress the proliferation of thyroid cancer cells [23]. In addition, research for malignant glioma, neuroblastoma and malignant peripheral nerve sheath tumors demonstrated that CDK4 copy number was amplified and was associated with tumor prognosis [23-25]. Moreover, our previous study of 514 cases of acral melanoma showed that $87 \%$ of patients had at least one copy number variant of the CDK4 signaling pathway genes. Cdk4 gain, Ccnd1 gain and the combination of Cdk4 gain and Ccnd1 gain were associated with the poor prognosis of acral melanoma, respectively [16]. In this study, copy number amplification of CDK4 and CCND1 genes and copy number deletion of CDKN2A gene were observed in most MM cases, suggesting that CDK4 signaling pathway aberrations maybe the driver for MM. Further analysis showed that CNV was significantly 

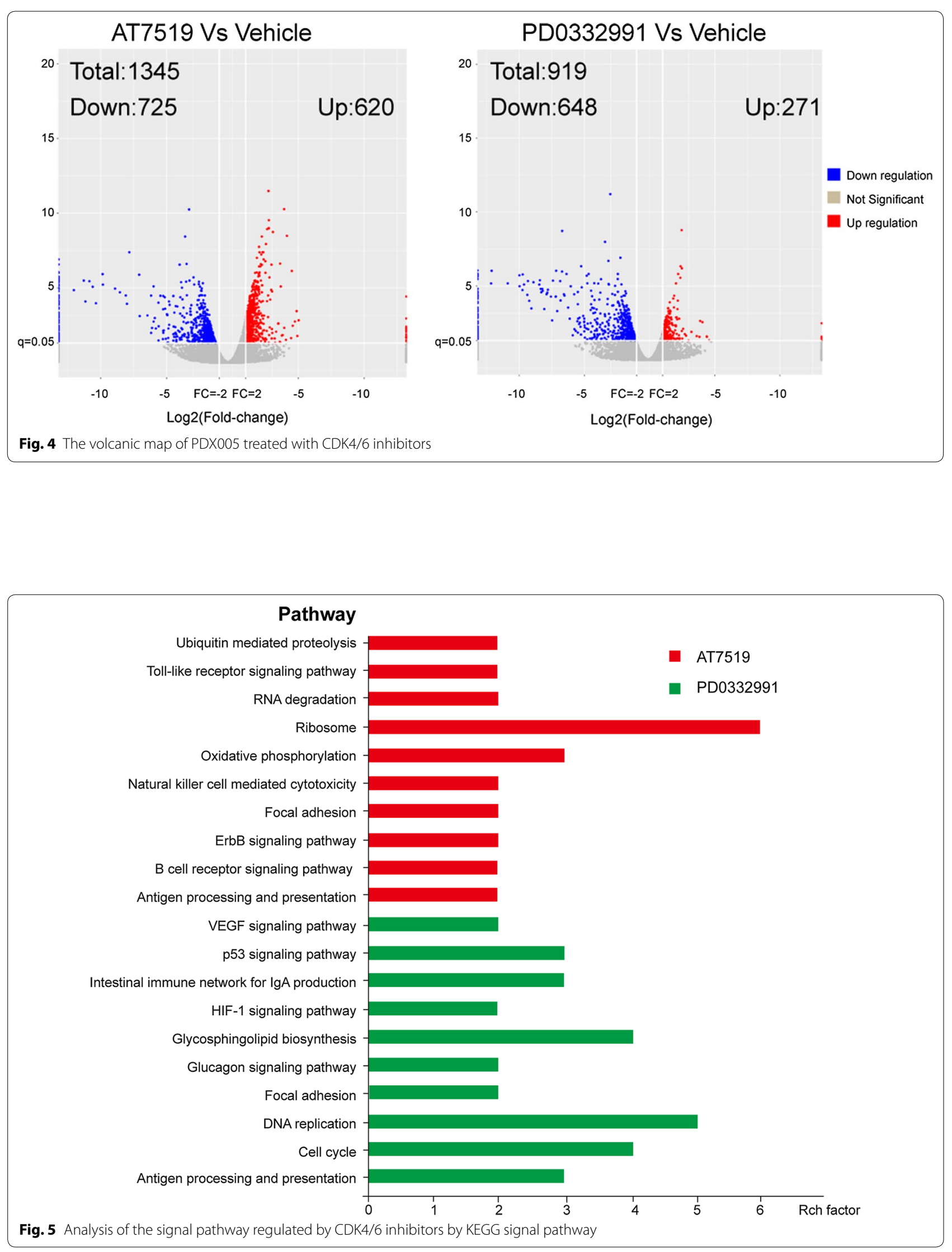
associated with the TNM stage in MM. However, the aberration of CDK4 signaling pathway genes was not significantly associated with the OS of MM patients. Therefore, it is necessary to expand the sample for further verification. Richard et al. found that CDKN2A copy number loss was a frequent event in patients with $\mathrm{CM}$, and the prognosis is worse in patients with increased Ccnd 1 copy number and Cdk4 copy number. However, after stratification of the status of NRAS and Braf mutations in patients, copy number variation in the CDK4 signaling pathway genes was not significantly associated with the survival of CM [26]. This is similar to our results because the mutation rates of NRAS and Braf is extremely low in MM patients, and the patients analyzed tend to be independent of NRAS and BRAF mutation.

Previous studies have shown that CDK inhibitors can inhibit the growth of a variety of tumors. CDK $4 / 6$ inhibitor Palbociclib combined with mTOR inhibitor MLN0128 suppresses the proliferation of ER negative breast cancer cells and Glioma [12, 27]. Similarly, triplenegative breast cancers with MT4-MMP, EGFR and RBpositive are sensitive to Erlotinib in combination with Palbociclib in PDX model [28]. Our previous study found that both CDK inhibitors and specific CDK4/6 inhibitors effectively inhibited the tumor growth in PDX models of acral melanoma containing CDK4 pathway aberration [16]. Zhou et al. found that CDK4 gene was amplified in $65 \mathrm{MM}$ patients and Palbociclib, an inhibitor of CDK4/6, effectively blunted the tumors harboring Cdk4 copy number gain in a PDX model [29]. Here, we found that both AT7519, a pan CDK inhibitor, and PD0332991, a selective CDK4/6 inhibitor, significantly inhibited the proliferation of MM cell lines in vitro. In vivo, both inhibitors obviously reduced the growth of tumors which harbored abnormal CDK4 signaling pathway in PDX models. However, tumors with normal CDK4 signaling pathway exhibited minimal sensitivity to both inhibitors. These results suggest that CDK4 aberration is an indicator for CDK4/6 inhibitors applied in MM. We also found in clinic that Palbociclib, an inhibitor of CDK4/6, can prolong the survival of MM patients with copy number variations of CDK4 pathway [30]. Therefore, CDK4 pathway genes copy number variation is a therapeutic target for MM.

To explore the potential molecular mechanisms, we subjected a PDX model (PDX-005) containing Cdk4 gain $+\mathrm{Ccnd} 1$ gain $+\mathrm{P} 16^{\mathrm{INK} 4 \mathrm{a}}$ loss to $\mathrm{RNA}$ sequencing. Analysis of GO and KEGG revealed significant changes in several signaling pathways, including cell cycle and immunity. It is worth noting that in the enrichment analysis of the biological process, cellular component, molecular function and KEGG Pathway, the immune-related signaling pathways were significantly altered during the four above-mentioned analyses. These results suggest that CDK4/6 inhibitors may play an important role in the development of MM by regulating the cellular immune signaling pathway. Alterations in immune-related signaling pathways suggest that CDK4/6 inhibitors may affect the cellular immune system, thus affecting the efficacy of immunotherapies to tumor. Immunotherapy, including PD-1 antibody, has revolutionized the treatment of many tumors. In breast cancer, Shom et al. found that CDK4/6 inhibitors not only induce tumor cell cycle arrest, but also promote anti-tumor immunity in two main way: 1 , CDK4/6 inhibitors alter tumor cell expression of endogenous retroviral components, hence increasing intracellular levels of double-stranded RNA. This in turn stimulates production of type III interferons and therefore enhances tumor antigen presentation. 2, CDK4/6 inhibitors markedly check the proliferation of regulatory $\mathrm{T}$ cells [31]. In general, the above-mentioned results suggest that CDK4/6 inhibitors are common in inducing antitumor immunity.

\section{Conclusion}

In this study, we found that the copy number of the components of CDK4 signaling pathway is altered in MM. Inhibition of CDK signaling pathway and CDK4 signaling pathway alone obviously suppresses the proliferation of MM cells and the tumor growth in PDX models harboring CDK4 pathway abnormity. This study provides theoretical basis for targeting CDK4 pathway in MM.

\section{Additional file}

Additional file 1: Table S1. Correlation of CDK4 pathway aberrations to treatment groups.

\section{Abbreviations}

AM: acral melanoma; bDNA: branched DNA; CM: cutaneous melanoma; CNV: copy number variation; FDR: false discovery rate; FFPE: Formalin-Fixed, Paraffin-Embedded Tissues; FPKM: Fragments Per Kilobase of exon model per Million mapped read; GO: gene ontology; KEGG: Kyoto Encyclopedia of Genes and Genomes; MM: mucosal melanoma; NOD/SCID: non-obese diabetic and severe combined immunodeficiency; OS: overall survival; PDX: patient-derived xenograft model; QGP: QuantiGenePlex DNA assay.

\section{Acknowledgements}

We thank the Dr. Zhiguo Zhao for English language editing.

\section{Authors' contributions}

$L X, Y K, J G$ designed the study. $L X, Z C, H Y$ performed the experiments. $L X, X W$ analyzed the data. $L X, Y K$ wrote the manuscript. All authors read and approved the final manuscript.

\section{Funding}

This work was supported by grants from Natural Science Foundation of China (81672696, 81772912), Fostering Young Scholars of Peking University Health Science Center,Clinical Medicine Plus X-Young Scholars Project (Peking University), the Fundamental Research Funds for the Central Universities, Beijing 
Baiqianwan Talents Project and Beijing Municipal Administration of Hospitals' Ascent Plan (No. DFL20181101)

\section{Availability of data and materials}

All the data and materials supporting the conclusion were included in the main paper.

\section{Ethics approval and consent to participate}

All procedures performed in studies involving human participants were in accordance with the ethical standards of the Medical Ethics Committee of the Beijing Cancer Hospital \& Institute and with the 1964 Helsinki declaration and its later amendments or comparable ethical standards. For this type of study formal consent is not required.

\section{Consent for publication}

Not applicable.

\section{Competing interests}

The authors declare that they have no competing interests.

Received: 27 May 2019 Accepted: 17 July 2019

Published online: 29 July 2019

\section{References}

1. Siegel RL, Miller KD, Jemal A. Cancer statistics, 2019. CA Cancer J Clin. 2019;69:7-34.

2. Chen W, Zheng R, Baade PD, Zhang S, Zeng H, Bray F, Jemal A, Yu XQ, He J. Cancer statistics in China, 2015. CA Cancer J Clin. 2016;66:115-32.

3. McLaughlin CC, Wu XC, Jemal A, Martin HJ, Roche LM, Chen VW. Incidence of noncutaneous melanomas in the U.S. Cancer. 2005;103:1000-7.

4. Chi Z, Li S, Sheng X, Si L, Cui C, Han M, Guo J. Clinical presentation, histology, and prognoses of malignant melanoma in ethnic Chinese: a study of 522 consecutive cases. BMC Cancer. 2011;11:85.

5. Lian B, Si L, Cui C, Chi Z, Sheng X, Mao L, Li S, Kong Y, Tang B, Guo J. Phase II randomized trial comparing high-dose IFN-alpha2b with temozolomide plus cisplatin as systemic adjuvant therapy for resected mucosal melanoma. Clin Cancer Res. 2013;19:4488-98.

6. Furney SJ, Turajlic S, Stamp G, Nohadani M, Carlisle A, Thomas JM, Hayes A, Strauss D, Gore M, van den Oord J, et al. Genome sequencing of mucosal melanomas reveals that they are driven by distinct mechanisms from cutaneous melanoma. J Pathol. 2013;230:261-9.

7. Krauthammer M, Kong Y, Ha BH, Evans P, Bacchiocchi A, McCusker JP, Cheng E, Davis MJ, Goh G, Choi M, et al. Exome sequencing identifies recurrent somatic RAC1 mutations in melanoma. Nat Genet. 2012;44:1006-14.

8. Curtin JA, Fridlyand J, Kageshita T, Patel HN, Busam KJ, Kutzner H, Cho KH, Aiba S, Brocker EB, LeBoit PE, et al. Distinct sets of genetic alterations in melanoma. N Engl J Med. 2005;353:2135-47.

9. Curtin JA, Busam K, Pinkel D, Bastian BC. Somatic activation of KIT in distinct subtypes of melanoma. J Clin Oncol. 2006;24:4340-6.

10. Flaherty KT, Puzanov I, Kim KB, Ribas A, McArthur GA, Sosman JA, O'Dwyer PJ, Lee RJ, Grippo JF, Nolop K, Chapman PB. Inhibition of mutated, activated BRAF in metastatic melanoma. N Engl J Med. 2010;363:809-19.

11. Hanahan D, Weinberg RA. Hallmarks of cancer: the next generation. Cell. 2011;144:646-74.

12. Yamamoto T, Kanaya N, Somlo G, Chen S. Synergistic anti-cancer activity of CDK4/6 inhibitor palbociclib and dual mTOR kinase inhibitor MLN0128 in pRb-expressing ER-negative breast cancer. Breast Cancer Res Treat. 2019;174(3):615-25.

13. Hsu CL, Lui KW, Chi LM, Kuo YC, Chao YK, Yeh CN, Lee LY, Huang Y, Lin TL, Huang MY, et al. Integrated genomic analyses in PDX model reveal a cyclin-dependent kinase inhibitor Palbociclib as a novel candidate drug for nasopharyngeal carcinoma. J Exp Clin Cancer Res. 2018;37:233.

14. Kwong LN, Costello JC, Liu H, Jiang S, Helms TL, Langsdorf AE, Jakubosky D, Genovese G, Muller FL, Jeong JH, et al. Oncogenic NRAS signaling differentially regulates survival and proliferation in melanoma. Nat Med. 2012;18:1503-10.
15. Hayes TK, Luo F, Cohen O, Goodale AB, Lee Y, Pantel S, Bagul M, Piccioni $F$, Root DE, Garraway LA, et al. A functional landscape of resistance to MEK $1 / 2$ and CDK4/6 inhibition in NRAS mutant melanoma. Cancer Res. 2019;79(9):2352-66.

16. Kong Y, Sheng X, Wu X, Yan J, Ma M, Yu J, Si L, Chi Z, Cui C, Dai J, et al. Frequent genetic aberrations in the CDK4 pathway in acral melanoma indicate the potential for CDK4/6 inhibitors in targeted therapy. Clin Cancer Res. 2017;23:6946-57.

17. Sheppard KE, McArthur GA. The cell-cycle regulator CDK4: an emerging therapeutic target in melanoma. Clin Cancer Res. 2013;19:5320-8.

18. Kong Y, Si L, Zhu Y, Xu X, Corless CL, Flaherty KT, Li L, Li H, Sheng X, Cui C, et al. Large-scale analysis of KIT aberrations in Chinese patients with melanoma. Clin Cancer Res. 2011;17:1684-91.

19. Si L, Kong Y, Xu X, Flaherty KT, Sheng X, Cui C, Chi Z, Li S, Mao L, Guo J. Prevalence of BRAF V600E mutation in Chinese melanoma patients: large scale analysis of BRAF and NRAS mutations in a 432-case cohort. Eur J Cancer. 2012;48:94-100.

20. Basudan A, Priedigkeit N, Hartmaier RJ, Sokol ES, Bahreini A, Watters RJ, Boisen MM, Bhargava R, Weiss KR, Karsten MM, et al. Frequent ESR1 and CDK pathway copy-number alterations in metastatic breast cancer. Mol Cancer Res. 2019;17:457-68.

21. Wang X, Kallionpaa RA, Gonzales PR, Chitale DA, Tousignant RN, Crowley JP, Chen Z, Yoder SJ, Blakeley JO, Acosta MT, et al. Germline and somatic NF1 alterations are linked to increased HER2 expression in breast cancer. Cancer Prev Res (Phila). 2018;11:655-64.

22. Ismail A, Bandla S, Reveiller M, Toia L, Zhou Z, Gooding WE, Kalatskaya I, Stein L, D'Souza M, Litle VR, et al. Early G(1) cyclin-dependent kinases as prognostic markers and potential therapeutic targets in esophageal adenocarcinoma. Clin Cancer Res. 2011;17:4513-22.

23. Valenciaga A, Saji M, Yu L, Zhang X, Bumrah C, Yilmaz AS, Knippler CM, Miles W, Giordano TJ, Cote GJ, Ringel MD. Transcriptional targeting of oncogene addiction in medullary thyroid cancer. JCI Insight. 2018;3:16.

24. Aoki K, Nakamura H, Suzuki H, Matsuo K, Kataoka K, Shimamura T, Motomura K, Ohka F, Shiina S, Yamamoto T, et al. Prognostic relevance of genetic alterations in diffuse lower-grade gliomas. Neuro Oncol. 2018:20:66-77.

25. Yu J, Deshmukh H, Payton JE, Dunham C, Scheithauer BW, Tihan T, Prayson RA, Guha A, Bridge JA, Ferner RE, et al. Array-based comparative genomic hybridization identifies CDK4 and FOXM1 alterations as independent predictors of survival in malignant peripheral nerve sheath tumor. Clin Cancer Res. 2011;17:1924-34.

26. Young RJ, Waldeck K, Martin C, Foo JH, Cameron DP, Kirby L, Do H, Mitchell C, Cullinane C, Liu W, et al. Loss of CDKN2A expression is a frequent event in primary invasive melanoma and correlates with sensitivity to the CDK4/6 inhibitor PD0332991 in melanoma cell lines. Pigment Cell Melanoma Res. 2014;27:590-600.

27. Olmez I, Brenneman B, Xiao A, Serbulea V, Benamar M, Zhang Y, Manigat L, Abbas T, Lee J, Nakano I, et al. Combined CDK4/6 and mTOR inhibition is synergistic against glioblastoma via multiple mechanisms. Clin Cancer Res. 2017;23:6958-68.

28. Foidart P, Yip C, Radermacher J, Blacher S, Lienard M, Montero-Ruiz L, Maquoi E, Montaudon E, Chateau-Joubert S, Collignon J, et al. Expression of MT4-MMP, EGFR, and RB in triple-negative breast cancer strongly sensitizes tumors to erlotinib and palbociclib combination therapy. Clin Cancer Res. 2018:25:1838-50.

29. Zhou R, Shi C, Tao W, Li J, Wu J, Han Y, Yang G, Gu Z, Xu S, Wang Y, et al. Analysis of mucosal melanoma whole-genome landscapes reveals clinically relevant genomic aberrations. Clin Cancer Res. 2019;25:3548-60.

30. Tang B, Sheng X, Kong Y, Chi Z, Si L, Cui C, Yan X, Mao L, Lian B, Li S, et al. Palbociclib for treatment of metastatic melanoma with copy number variations of CDK4 pathway: case report. Chin Clin Oncol. 2018;7:62.

31. Goel S, DeCristo MJ, Watt AC, BrinJones H, Sceneay J, Li BB, Khan N, Ubellacker JM, Xie S, Metzger-Filho O, et al. CDK4/6 inhibition triggers anti-tumour immunity. Nature. 2017;548:471-5.

\section{Publisher's Note}

Springer Nature remains neutral with regard to jurisdictional claims in published maps and institutional affiliations. 